\title{
Establishing sentinel lymph node as a standard of care in gynecologic cancer?
}

In this series of the journal, the topics lectures given at the first IRCAD course on sentinel lymph node (SLN) in gynecologic oncology held in Strasbourg (France) in 2019 are compiled in order to address all the aspects of the theoretical basis and clinical applications of a concept which is becoming universally popular in gynecologic oncology.

SLN is an attractive evolution of a mainstay of gynecologic oncology and in surgical oncology in general, which is lymph node staging. Lymph node dissection has been for long the only way to comprehensively and accurately stage gynecologic cancers, however with scarce evidence of impact on survival outcomes. In addition to knowledge of the occurrence of complication, the increasing awareness of long term side effects like lymphoedema has led to question the clinical relevance of systematic staging. Interestingly, the SLN concept refers to natural history of lymphatic spread of cancer, yet in diametrical opposition with the Halstedian concept, by which all potential sites of tumor spread had to be removed. Vulvar cancer has been the first validated application, fueled by the high morbidity of groin dissection. In cervical cancer, in spite of the fact that lymph node staging cannot be at this point in time limited to sentinel nodes, the technique marks the potential end of the obsession to miss diseased nodes. In endometrial cancer, the universal implementation of the technique is likely to close the controversy between believers and non-believers in lymph node dissection.

Even before becoming a standard, the SLN technique has impacted the template of lymph node dissection. In vulvar cancers, attention has to be drawn to the frequent localization of the most important node in the lateral prepubic area, a location potentially missed by the traditional groin dissection of the Scarpa triangle, which in addition removes some nodes, the most caudal ones, which are not related to the vulva area. In cervical cancer, the existence of pathways different from the most frequently observed (the obturator/internal iliac pathway), has made mandatory to include the common iliac area and the presacral area in the standard pelvic lymph node dissection, in contrast with the policy followed by many centers, in which these locations were neglected. The virtual absence of primary metastases in the circumflex iliac nodes, the most caudal ones of the external iliac area, has been confirmed. Omitting the removal of the latter nodes has the potential to substantially decrease the rate of lymphoedema. The same is true in endometrial cancer. Finally, in all tumor sites, the implementation of the SLN procedure makes the surgeon more confident not that he does not leave a diseased node behind.

It cannot however be ignored that the SLN procedures may be made less accurate and even dangerous as a result of two categories of pitfalls, one related to surgery, the other one to pathology.

Surgically speaking, there is a definite risk of misusing the concept by applying a faulty technique as a reason of inexperience. Inadequate injection site, removal of the second/third level node, removal of empty packets not containing nodes, are associated with early steps of learning curve. Even if the initial tracers, blue dyes and technetium, are still accepted, it is becoming obvious that the technique is less accurate when the most recent infra-red fluorescence technique is not available. Finally, the identification of lymph nodes must follow a simple but strict algorithm by which all clinically suspicious must be removed even if not injected, all nodes of a given area must be removed if no SLN is detected, and all removed nodes must be sent to the pathologist for ultrastaging.

Pathology is a key component of the diagnostic chain. Close collaboration with a pathologist fully informed of the objectives and consequences of the procedure is mandatory. Meticulous macroscopic and microscopic examination of the node, with serial sections is necessary. There are controversies regarding the benefit of frozen section, which may miss low volume disease while destroying part of the material. On the other hand, the comprehensive assessment of the nodes has resulted in a substantial increase of a finding almost unknown until recently: micrometastases and isolated tumor cells. As the prognostic value and therapeutic impact of the finding of low volume disease is not completely clarified, the clinician may be in trouble when deciding on adjuvant treatment. Finally, discovering isolated nodes in the parametrial in uterine cancer may be misinterpreted as stage IIIC, while it still assigns to stage IIB.

In spite of these reservations, SLN has become one of the emblematic techniques which define the gynecologic oncologist, has the potential of reducing the long-term effects of full lymph node dissection, may spare the patient unnecessary radical hysterectomy when nodal disease is found intraoperatively, improves the precision of staging, is universally accepted as staging 
of early vulvar cancer, and can already be used in case of unresolved controversy about the need of lymph node dissection.

In the future, if sentinel node only is finally accepted as the mainstay of nodal staging, a breast/cutaneous melanoma model may be implemented, by which the adjuvant treatment is decided on the basis of pathologic findings in the SLN. The surgical management of early cervical cancer will be preceded by minimally invasive SLN procedure with permanent pathology as preoperative workup, a move already implemented in vulvar cancer. Awaiting this, the mainstay of surgical management of stage I endometrial cancers is more and more evolving towards the combination of hysterectomy and SLN.

\section{Acknowledgments}

Funding: None.

\section{Footnote}

Provenance and Peer Review: This article was commissioned by the editorial office, Chinese Clinical Oncology, for the series "Sentinel Lymph Node Biopsy in Gynecologic Cancer". The article did not undergo external peer review.

Conflicts of Interest: Both authors have completed the ICMJE uniform disclosure form (available at http://dx.doi.org/10.21037/ cco-21-11). The series "Sentinel Lymph Node Biopsy in Gynecologic Cancer" was commissioned by the editorial office without any funding or sponsorship. Drs. DQ and CA served as the unpaid Guest Editors of the series. Dr. DQ serves as an unpaid editorial board member of Chinese Clinical Oncology from Dec 2019 to Dec 2021. The authors have no other conflicts of interest to declare.

Ethical Statement: The authors are accountable for all aspects of the work in ensuring that questions related to the accuracy or integrity of any part of the work are appropriately investigated and resolved.

Open Access Statement: This is an Open Access article distributed in accordance with the Creative Commons AttributionNonCommercial-NoDerivs 4.0 International License (CC BY-NC-ND 4.0), which permits the non-commercial replication and distribution of the article with the strict proviso that no changes or edits are made and the original work is properly cited (including links to both the formal publication through the relevant DOI and the license). See: https://creativecommons.org/ licenses/by-nc-nd/4.0/.

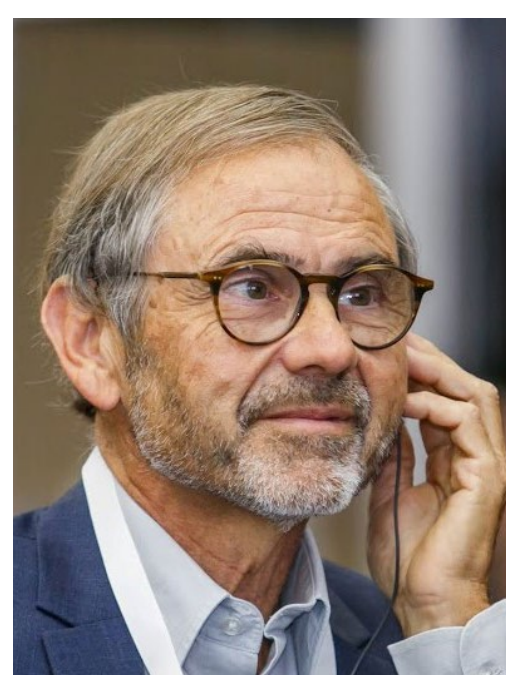

Denis Querleu

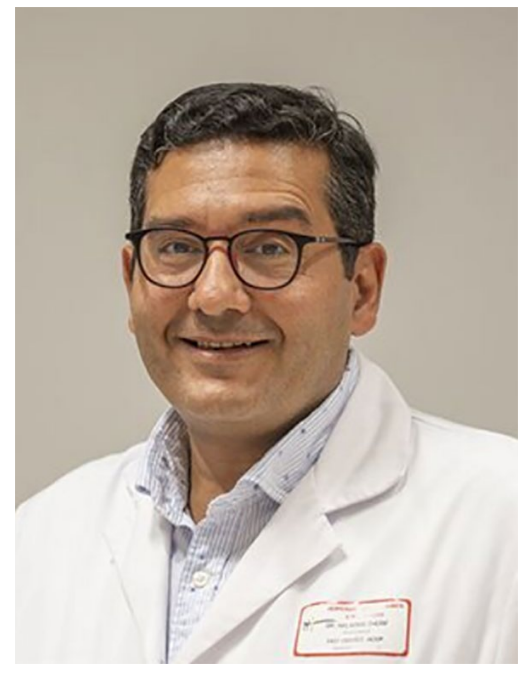

Chérif Akladios 


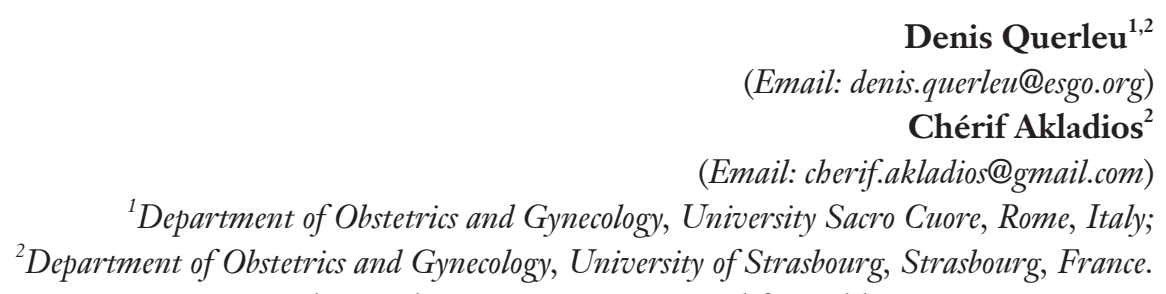

Submitted Jan 22, 2021. Accepted for publication Jan 27, 2021. doi: $10.21037 / \mathrm{cco}-21-11$

View this article at: http://dx.doi.org/10.21037/cco-21-11

Cite this article as: Querleu D, Akladios C. Establishing sentinel lymph node as a standard of care in gynecologic cancer? Chin Clin Oncol 2021;10(2):13. doi: 10.21037/cco-21-11 